\title{
Mathematics of the NFAT signalling pathway
}

\author{
Alan D. Rendall \\ Max-Planck-Institut für Gravitationsphysik \\ Albert-Einstein-Institut \\ Am Mühlenberg 1 \\ 14476 Potsdam, Germany
}

\begin{abstract}
This paper is a mathematical study of some aspects of the signalling pathway leading to the activation of the transcription factor NFAT (nuclear factor of activated $\mathrm{T}$ cells). Activation takes place by dephosphorylation at multiple sites. This has been modelled by Salazar and Höfer using a large system of ordinary differential equations depending on many parameters. With the help of chemical reaction network theory we show that for any choice of the parameters this system has a unique stationary solution for each value of the conserved quantity given by the total amount of NFAT and that all solutions converge to this stationary solution at late times. The dephosphorylation is carried out by calcineurin, which in turn is activated by a rise in calcium concentration. We study the way in which the dynamics of the calcium concentration influences NFAT activation, an issue also considered by Salazar and Höfer with the help of a model arising from work of Somogyi and Stucki. Criteria are obtained for convergence to equilibrium of solutions of the model for the calcium concentration.
\end{abstract}

\section{Introduction}

The phenomena modelled mathematically in this paper are mechanisms which are part of the way the immune system works at the molecular level. For background on immunology the reader is referred to [17] or [21]. T cells are among the most important components of the immune system. They have the task of recognizing certain antigens and reacting appropriately. More precisely, a $\mathrm{T}$ cell recognizes a peptide (small protein) in combination with an MHC (major histocompatibility complex) molecule. The recognition takes place through a surface molecule, the $\mathrm{T}$ cell receptor. In what follows attention will be confined to $\mathrm{T}$ helper (Th) cells although some of the statements made may also apply to other types of $\mathrm{T}$ cells. In order for the $\mathrm{T}$ cell to be activated a second signal is also necessary. This comes from another surface molecule, CD28, which recognizes the molecules $\mathrm{B} 7.1$ and $\mathrm{B} 7.2$ on the antigen presenting cell carrying the peptide-MHC complex. The information about these recognition events 
is propagated to the nucleus through various signalling pathways. The result is that the transcription factors NFAT, $\mathrm{NF} \kappa \mathrm{B}$ and $\mathrm{AP}-1$ bind to the DNA, leading to the production of the cytokine IL-2 (interleukin 2). Much remains to be learned about these signalling pathways and mathematical modelling has a great potential to contribute to obtaining a better understanding of them.

In the following attention will be concentrated on the part of the signalling network relating to NFAT (nuclear factor of activated $\mathrm{T}$ cells). It should be noted that although the abbreviation NFAT refers to $\mathrm{T}$ cells this transcription factor is important for signalling in many other types of cells. There are five different NFAT molecules and the one of relevance in what follows is that known as NFATc2 or NFAT1. A model for NFAT signalling in T cells was introduced by Salazar and Höfer 22. In fact they only deal with part of the pathway. An important stage in signalling is when there is a flow of calcium ions into the cytosol. During the activation of $\mathrm{T}$ cells this occurs when $\mathrm{IP}_{3}$ (inositol 1,4,5trisphosphate) binds to receptors in the endoplasmic reticulum (ER), opening calcium channels and thus allowing calcium ions to flow down their concentration gradient. This can be simulated experimentally by treating the cells with ionomycin, which leads to transport of calcium ions across membranes. The first step in the NFAT pathway included in the work of [22] and the first one to play a role in what follows, is this increase in the calcium concentration. The calcium binds to calcineurin, partly activating it. It also binds to calmodulin, which can then complete the activation of calcineurin. The activated calcineurin removes phosphate groups from NFAT, which is present in phosphorylated form in the cytosol of resting cells. The NFAT then undergoes a conformational change and moves to the nucleus where it can bind to DNA. The main model in 22] (which will be called the SH model in what follows) describes the dephosphorylation of NFAT and its transport between the cytosol and the nucleus. A subsidiary model describes the calcium influx. The aim of this paper is to obtain a deeper mathematical understanding of these models.

The SH model is a system of $4 N+4$ equations and contains $10 N+4$ parameters, where $N$ is the number of phosphorylation sites. The case of interest for NFAT is $N=13$ so that there are 56 equations and 134 parameters. Due to the large number of variables involved it might seem difficult to analyse the dynamical behaviour of general solutions of this system. Chemical reaction network theory (CRNT) 9] is a general tool for attacking this type of problem and it turns out to be very effective in this case. As we will show, one of its strongest theorems, the Deficiency Zero Theorem, can be applied to this system. The result is that for a given total amount of NFAT there is a unique stationary solution of the system and that every other solution converges to the stationary solution.

The SH model describes the dephosphorylation of NFAT when the concentration of activated calcineurin is constant. In fact the calcium influx which leads to the activation of NFAT is a dynamical process. To assess the applicability of the SH model it is desirable to know whether the calcium concentration tends to a constant value at late times. This process is modelled in 22] by a two-dimensional dynamical system. It will be shown that for certain subsets 
of the parameter space for this model the solutions do converge to a stationary solution. It is also shown that when this happens the long-time behaviour of the amounts of the different forms of NFAT occurring in the SH model is that they converge to the values they converge to in the SH model with an appropriate choice of parameters.

The paper is organized as follows. Section 2 contains some basic material about chemical reaction network theory. The dynamical analysis of the SH model for NFAT phosphorylation with constant stimulation is in section 3, The dynamics of the calcium influx is investigated in section 4. The last section of the main text gives conclusions and an outlook. In an appendix the SH model is compared with a model for the NFAT signalling pathway defined in [10].

\section{Chemical reaction network theory}

Chemical reaction network theory is a collection of methods for studying the dynamics of solutions of ordinary differential equations modelling systems of chemical reactions. Some concepts of this theory will now be reviewed. In CRNT the basic objects are finite sets $\mathcal{S}$ of species, $\mathcal{C}$ of complexes and $\mathcal{R}$ of reactions. The elements of $\mathcal{C}$ are formal linear combinations of elements of $\mathcal{S}$ with positive integer coefficients while the elements of $\mathcal{R}$ are ordered pairs of elements of $\mathcal{C}$. The number of elements of $\mathcal{S}, \mathcal{C}$ and $\mathcal{R}$ are denoted by $m, \bar{n}$ and $r$ respectively. The set $\mathcal{S}$ consists of the substances taking part in the chemical reactions and in the example of the SH system it consists of $4(N+1)$ states of NFAT. The complexes are the combinations of species occurring on the left and right hand sides of the reactions. In the SH model each complex is just a single species. The reactions are ordered pairs of complexes, each representing the input and output of one reaction. In the case of the $\mathrm{SH}$ model they can be identified with ordered pairs of species. (Note that a reaction and the reverse reaction are counted separately.) Given a reaction network let $c_{s}$ be the concentration of the species with index $s$. Consider a system of ordinary differential equations of the form

$$
\frac{d c_{s}}{d t}=f_{s}(y)=\sum_{\left(y, y^{\prime}\right) \in \mathcal{R}} r\left(y, y^{\prime}\right)\left(y_{s}^{\prime}-y_{s}\right) .
$$

Here $r\left(y, y^{\prime}\right) \geq 0$ are the reaction rates. They are assumed non-negative. The choice of these functions is often referred to as the kinetics. In this section only the most standard choice of kinetics will be considered. This is mass-action kinetics, where

$$
r\left(y, y^{\prime}\right)=k_{y y^{\prime}} c^{y} .
$$

Here $k_{y y^{\prime}}$ are positive constants called the rate constants and $c^{y}=\prod_{s \in \mathcal{S}} c_{s}^{y_{s}}$. The equation (11) will be abbreviated to $\dot{c}=f(c)$. Here $c$ is a vector of concentrations $c_{s}$ and so is a point of $\mathbf{R}^{m}$. The positive and non-negative orthants are defined to be the sets of points of $\mathbf{R}^{m}$ whose coordinates are positive and non-negative, respectively. The quantity $c$ is said to be positive (non-negative) 
if it lies in the positive (non-negative) orthant. Because of its interpretation in terms of concentrations $c$ should be non-negative in order to be of relevance for applications.

The positive and non-negative orthants are invariant under the evolution defined by the ordinary differential equations of a chemical reaction network. The invariance of the non-negative orthant follows from that of the positive orthant by continuity. The invariance of the positive orthant is a consequence of a lemma which will now be proved. (Cf. Lemma II.1 of [25] for a similar result.)

Lemma 1 Consider a solution $c_{s}(t)$ of (1) with the coefficients $r\left(y, y^{\prime}\right)$ being given by mass-action kinetics. If $c_{s}\left(t_{0}\right)>0$ for some $s$ and some time $t_{0}$ then $c_{s}(t)>0$ for all $t \geq t_{0}$ for which the solution exists.

Proof If the statement of the lemma is false then it can be assumed that $c_{s}\left(t_{1}\right)=$ 0 for some $t_{1}>t_{0}$. The time $t_{1}$ can be chosen so that $c_{s}(t)>0$ for all $t<t_{1}$. The quantity $c_{s}$ satisfies an equation of the form

$$
\frac{d c_{s}}{d t}=-f_{-}(c) c_{s}+f_{+}(c)
$$

where $f_{+}$is non-negative. Since $c_{s}$ is positive on the interval $\left[t_{0}, t_{1}\right)$ the inequality

$$
\frac{d}{d t}\left(\log c_{s}\right) \geq-f_{-}(c)
$$

holds. Integrating this equation and exponentiating gives

$$
c_{s}\left(t_{1}\right) \geq c_{s}\left(t_{0}\right) \exp \left(-\int_{t_{0}}^{t_{1}}\left|f_{-}(c(t))\right| d t\right) .
$$

The integral in this expression is finite and so the inequality implies that $c_{s}\left(t_{1}\right)>$ 0 , a contradiction. This completes the proof of the lemma.

The reaction network can be represented as a directed graph where the vertices are the complexes and edges represent reactions. The reaction network is said to be weakly reversible if whenever it is possible to link the complex $y$ to the complex $y^{\prime}$ by a sequence of reactions it is also possible to link $y^{\prime}$ to $y$ in the same way. In the case of the SH system the network is weakly reversible since in fact every reaction is reversible. The connected components of the reaction graph are called linkage classes and their number is denoted by $l$. In the case of the SH model $l=1$. An important object is the stoichiometric matrix $\bar{N}$. Its columns correspond to the reactions belonging to the network. The entries in a column are defined by the sums of coefficients of the different species in the complexes occurring in the reaction, with the coefficients on the left hand side being counted negatively and the coefficients on the right hand side positively. In other words, these are the net number of molecules of each species produced in the reaction. It is an $m \times r$ matrix. The cosets of the form $c+i m \bar{N}$ are called stoichiometric compatibility classes and are invariant under the flow of the system. The intersection of a stoichiometric compatibility class with the 
non-negative orthant is called a reaction simplex and is also invariant under the flow by Lemma 1 . The rank of $\bar{N}$ (i.e. the dimension of the stoichiometric compatibility classes) is denoted by $s$. The deficiency of the network is defined by $\delta=\bar{n}-l-s$. The following is part of the Deficiency Zero Theorem which was first proved in [14, 15] and [8].

Theorem 1 Let $\dot{c}=f(c)$ be the system of ordinary differential equations defined by a chemical reaction network by means of mass-action kinetics. If the network is weakly reversible and of deficiency zero then there is a unique positive stationary solution in each stoichiometric compatibility class. The solution is asymptotically stable within its class.

An important part of the proof of Theorem 1 is to show that there exists a Lyapunov function $L(c)$ which is non-increasing along solutions and strictly decreasing along all positive solutions except for the stationary solution. This means that the stationary solution is the only possible positive $\omega$-limit point of a positive solution. The function $f$ can be written in the form $Y g$ where $Y$ is called the complex matrix. Its columns are in one to one correspondence with the complexes and the entries in the column corresponding to the complex $y$ are the components $y_{s}$. In the case of the $\mathrm{SH}$ system $Y$ is just the identity. If $c_{*}$ is a stationary solution then $f\left(c_{*}\right)=0$. If in addition $g\left(c_{*}\right)=0$ the stationary solution is called complex balanced. Evidently any stationary solution of the SH system is complex balanced. In fact it is a consequence of the proof of Theorem 1 that for a system satisfying the assumptions of that theorem the intersection of the kernel of $Y$ with the image of $\bar{N}$ is $\{0\}$, so that any stationary solution of a system of that type is complex balanced.

\section{The model for NFAT phosphorylation}

The basic variables in the model of [22] are amounts of different forms of NFAT. Using amounts rather than concentrations avoids introducing extra factors of the ratio of the volumes of the two compartments. This is just a matter of mathematical convenience. Phosphate groups can be attached to this molecule at up to $N=13$ sites. The index $n$ will be used to denote the number of phosphate groups and runs from zero to $N$. It is assumed that the phosphate groups are bound to the sites in a certain order and are removed in the reverse order. There are thus $N$ phosphorylation states in total. Each of these has an active and an inactive form. Each of them occurs in the cytosol and in the nucleus. This gives a total of $4(N+1)$ variables decribing the amounts of the different substances. The processes of attaching a phosphate group and the conformational change between the active and inactive forms are reversible. It is necessary to prescribe $6 N+2$ rate constants to describe the reactions in a given compartment. However the rate constants describing the transitions between active and inactive forms are chosen to be the same in both compartments. Rate constants are also required to describe the transport processes between the two

compartments - the active form is transported into the nucleus and the inactive form out of the nucleus. It is assumed that there is just one rate constant 
for each of these two processes, independent of the phosphorylation state. A diagram of this reaction network can be found in [22, Fig. 1. Assuming massaction kinetics leads to a system of ordinary differential equations for the time evolution of the amounts of the different substances.

The unknowns are as follows. The amount of active NFAT in the cytoplasm with $n$ phosphorylated residues is denoted by $a_{n}, n=0,1, \ldots, N$. The amount of the corresponding inactive form is denoted by $i_{n}$. The amounts of these substances in the nucleus are denoted by $A_{n}$ and $I_{n}$. All these quantities are supposed non-negative. Mass-action kinetics is assumed. For $1 \leq n \leq N-1$ the dynamical equations for amounts in the nucleus are

$$
\begin{aligned}
& \frac{d A_{n}}{d t}=K_{n-1} A_{n-1}-K_{n} A_{n}+C_{n} A_{n+1}-C_{n-1} A_{n} \\
& +l_{n}^{-} I_{n}-l_{n}^{+} A_{n}+d a_{n}, \\
& \frac{d I_{n}}{d t}=K_{n-1}^{\prime} I_{n-1}-K_{n}^{\prime} I_{n}+C_{n}^{\prime} I_{n+1}-C_{n-1}^{\prime} I_{n} \\
& +l_{n}^{+} A_{n}-l_{n}^{-} I_{n}-f I_{n}
\end{aligned}
$$

with rate constants $K_{n}, K_{n}^{\prime}, C_{n}, C_{n}^{\prime}, l_{n}^{+}, l_{n}^{-}, d$ and $f$. Evolution equations for the cases $n=0$ and $n=N$ can be obtained by taking equations formally identical to those above with the conventions that $K_{-1}=C_{-1}=K_{-1}^{\prime}=C_{-1}^{\prime}=0$ and that $C_{N}=C_{N}^{\prime}=K_{N}=K_{N}^{\prime}=0$. Analogous evolution equations for the quantities $a_{n}$ and $i_{n}$ can be obtained as follows. Replace $A_{n}$ and $I_{n}$ by $a_{n}$ and $i_{n}$ respectively everywhere except in the last term of each equation. Reverse the sign in the last term of each equation. Replace $C_{n}, C_{n}^{\prime}, K_{n}, K_{n}^{\prime}$ by $c_{n}, c_{n}^{\prime}, k_{n}$ and $k_{n}^{\prime}$. It is stated in [22] that the transport processes are much slower than the reactions within each compartment. On a heuristic level this can be imported into the mathematics by assuming that the coefficients $d$ and $f$ are very small. It may be hoped that solutions of the full system can be approximated by solutions of the system obtained by setting $d=f=0$. In the latter system the equations describing amounts in the nucleus and the cytoplasm decouple. For this reason it is referred to in what follows as the decoupled system. To analyse this system it is enough to analyse the subsystems describing the dynamics in each compartment.

The SH system satisfies $\bar{n}=4(N+1)$ and $l=1$. In order to show that Theorem 1 applies it therefore suffices to show that the rank of $\bar{N}$ is $4 N+3$. This rank is equal to $4(N+1)$ minus the dimension of the kernel of $(\bar{N})^{T}$. The conditions for a vector to lie in this kernel are easy to analyse. Some of the conditions imply that all the components of the vector corresponding to amounts of substances in the cytosol are equal and that those corresponding to amounts of substances in the nucleus are equal. Finally elements corresponding to the amounts of the same substance in both compartments are equal. Thus the kernel is one-dimensional and the rank of the stoichiometric matrix is $4 N+3$. It follows that the deficiency is zero and Theorem 1 applies to the SH system.

Theorem 1 leaves open the question whether under its hypotheses every solution converges to a stationary solution. It will be shown below that this is 
the case for the SH system. Note first that the total amount of NFAT, which is the sum of all the variables in the SH system, is a conserved quantity. Thus each reaction simplex is compact. It follows that all solutions exist globally in time towards the future and the $\omega$-limit set of any positive solution is connected. Combining this with the remark about $\omega$-limit points made previously shows that if a solution does not converge to the stationary solution its $\omega$-limit set must be contained in the boundary of the positive orthant.

Suppose now that a positive solution $c$ has an $\omega$-limit point in the boundary of the positive orthant. There is a solution of the system, say $c_{\infty}$, passing through that $\omega$-limit point. The range of $c_{\infty}$ is contained entirely in the boundary. By Lemma 1 the number of non-zero components of $c_{\infty}$ can never decrease. It might a priori increase but in any case it will be constant after a finite time. Thus when considering late-time behaviour it may be assumed without loss of generality than $\left(c_{\infty}\right)_{s}(t)$ is non-zero for $1 \leq s \leq k$ and identically zero for $k+1 \leq s \leq m$. Here $0 \leq k<m$. In fact $k>0$ since the sum of the variables $\left(c_{\infty}\right)_{s}$, which is the total amount of NFAT in the cell, is conserved. If $k+1 \leq s \leq m$ then $\left(\dot{c}_{\infty}\right)_{s}=0$. There are no negative contributions to $\left(\dot{c}_{\infty}\right)_{s}$ since reactions having species $s$ on their left hand side are not active. If there is a link from species $s$ to species $s^{\prime}$ in the reaction network with non-zero concentration then a positive contribution to $\left(\dot{c}_{\infty}\right)_{s}$ results. It follows that if $s^{\prime}$ is adjacent to $s$ in the network then $k+1 \leq s^{\prime} \leq m$. Since the reaction graph is connected this implies that all $c_{s}$ vanish identically, a contradiction. Thus it has been proved that there can be no $\omega$-limit points on the boundary and the following result is obtained:

Theorem 2 Let $\dot{c}=f(c)$ be the system of Salazar and Höfer. There is a unique stationary solution $c_{*}$ in each stoichiometric compatibility class and each positive solution converges to a stationary solution as $t \rightarrow \infty$.

It has been conjectured that under the hypotheses of Theorem 1 every solution converges to a stationary solution as $t \rightarrow \infty$. This is known as the global attractor conjecture 4 and has recently been proved in the case that there is only one linkage class by Anderson [2]. Theorem 2 could be deduced from the result of [2] but it has been shown here that there is a much easier proof in this relatively simple case.

There are many different ways in which multiple phosphorylation can be organized and this can give rise to many systems related to the SH model. The phosphorylation is said to be processive if an enzyme which binds its substrate once phosphorylates several sites before dissociating. It is said to be distributive if only one site per binding event is phosphorylated. One type of distributive phosphorylation is sequential phosphorylation, where the sites are phosphorylated in a particular order and dephosphorylated in the reverse order - this is the case in the SH model. There is also a cyclic variant where dephosphorylation takes place in the same order as phosphorylation. It is also possible to consider phosphorylation in a random order or mixtures of the mechanisms introduced. An extensive discussion of the possibilities and examples of biological systems where they occur can be found in [23]. For many of these systems an analogue 
of Theorem 2 holds and can be proved in a similar way. For the only properties required for the proof are as follows:

- each complex consists of one species

- there is only one linkage class

- the network is weakly reversible

Thus the analogues of the SH model with cyclic or random phosphorylation both have the property that there is a unique stationary solution in each stoichiometric compatibility class and that any other solution converges to a stationary solution.

There is another dynamical system related to the modelling of $\mathrm{T}$ cell activation which has deficiency zero and can thus be shown to have the property that any solution converges to a stationary solution and that there is only one stationary solution in any stoichiometric compatibility class. This is the kinetic proofreading model of McKeithan [16] for antigen recognition by the T cell receptor and its dynamics was analysed mathematically by Sontag [25]. In that paper the analogue of Theorem 2 is proved for McKeithan's model. The key observation is that the deficiency of the network is zero so that Theorem 1 applies. From there it is possible to obtain the analogue of Theorem 2 for that system in a way very similar to what has just been done for the SH model.

In the SH model each phosphorylation or dephosphorylation is modelled as a single reaction and the details of the interaction with the enzyme which catalyses the process are not included. Suppose that instead the enzyme is incorporated in the standard Michaelis-Menten way [18. This means that the reactions describing the formation of a complex of the substrate with the enzyme and the dissociation of the complex to give either enzyme and substrate or enzyme and product are included, using mass-action kinetics. This is what is referred to as Michaelis-Menten via mass action (MMvma) in [1] and is different from using an effective Michaelis-Menten kinetics for a single reaction. The analogue of one of the parts of the decoupled SH model with the simple mass-action kinetics replaced by MMvma kinetics is similar to what is called a multiple futile cycle in [26]. In that case there is only one kinase which catalyzes all phosphorylations and one phosphatase which catalyzes all dephosphorylations. This is slightly different from the situation in 11], where there is a different enzyme for each reaction. In [26] upper and lower bounds for the number of stationary solutions of a system of this type are obtained. It follows from these that while in the case $N=1$ there is only one stationary solution there are at least three stationary solutions for $N=2$ and at least thirteen for $N=13$ for suitable choices of the parameters of the system. This corresponds to the case where the total concentrations of the enzymes are small compared to the total concentration of the substrates. The number of stationary solutions is never greater than $2 N-1$, whatever the parameters. If the total concentrations of the enzymes are sufficiently large compared to the total concentrations of the substrates then there is at most one stationary solution. 
The equations arising in the decoupled system can be analysed in the same way as the full system and the analogue of Theorem 2 holds in that case. For the decoupled system more can be done and the stationary solutions can be calculated explicitly, as was shown in [22. They are obtained by setting the sum of certain pairs of terms to zero. In the terminology of CRNT these stationary solutions are detailed balanced. Whether this gives the most general stationary solutions of the decoupled system is not discussed in 22 but it follows from the analogue of Theorem 2 that they are. To be concrete the system describing concentrations in the cytosol will be considered. To obtain the class of solutions found in 22] it is assumed that the first two terms on the second line of the evolution equation for $a_{n}$ cancel. This also gives a similar cancellation in the evolution equation for $i_{n}$. The condition for this is that $\frac{i_{n}}{a_{n}}=L_{n}$ where $L_{n}=\frac{l_{n}^{-}}{l_{n}^{+}}$. Next it is assumed that the second and third terms in the evolution equations for $a_{n}$ cancel for $0 \leq n \leq N-1$, giving

$$
\frac{a_{n+1}}{a_{n}}=\frac{k_{n}}{c_{n}} .
$$

Then the first and fourth terms cancel except in the case $n=0$. In fact, if the equations involving $L_{n}$ are satisfied and the equations (8) are satisfied for all $n \leq N-1$ then the conditions for a stationary solution of the part of the decoupled system describing concentrations in the cytosol is satisfied. Note that these detailed balance conditions cannot be satisfied by a stationary solution of the full system with non-zero coefficients $d$ and $f$. They may, however, be approximately satisfied when $d$ and $f$ are small. For these stationary solutions of the decoupled system the fraction of the NFAT in the cytosol which is in the active state can be computed. It is given by

$$
\phi=\frac{\sum_{n=0}^{N} a_{n}}{\sum_{n=0}^{N}\left(a_{n}+i_{n}\right)}=\frac{1+\sum_{n=1}^{N}\left(\prod_{j=0}^{n-1} \frac{k_{j}}{c_{j}}\right)}{1+L_{0}+\sum_{n=1}^{N}\left(1+L_{n}\right) \prod_{j=0}^{n-1} \frac{k_{j}}{c_{j}}} .
$$

In order to have a better understanding of the system it is useful to consider a special case with a reduced number of parameters. This is obtained in the following way. The coefficients $k_{n}, k_{n}^{\prime}, c_{n}$ and $c_{n}^{\prime}$ are taken to be independent of $n$ and denoted by $k, k^{\prime}, c$ and $c^{\prime}$ respectively. It is also assumed that $L_{n}=L_{0} \lambda^{n}$. In this case the expression for $\phi$ becomes

$$
\phi=\frac{\sum_{n=0}^{N}\left(\frac{k}{c}\right)^{n}}{\sum_{n=0}^{N}\left(1+L_{0} \lambda^{n}\right)\left(\frac{k}{c}\right)^{n}}=\left[1+L_{0} \frac{\left(\frac{\lambda k}{c}\right)^{N+1}-1}{\frac{\lambda k}{c}-1} \frac{\frac{k}{c}-1}{\left(\frac{k}{c}\right)^{N+1}-1}\right]^{-1} .
$$

One of the results of 22 is that for large $N$ the function $\phi$ resembles a Hill function $\phi_{H}(c)=\frac{c^{N}}{A+c^{N}}$ for a constant $A$ and an exponent $N$. In what sense does this resemblance hold? If $N$ is allowed to tend to infinity for fixed $A$ then the Hill function tends pointwise almost everywhere to a translated Heaviside function which is zero for $c<1$ and one for $c>1$. From the point of view of the 
applications this gives rise to a switch behaviour for large $N$. For values of the control parameter $c$ smaller than a threshold almost all the NFAT in the cytosol is in the inactive form while for values larger than the threshold almost all the NFAT is in the active form. The limiting behaviour of the function (10) as $N$ tends to infinity depends on the assumptions made about the other parameters present. If the other parameters are fixed then what is obtained in the limit does contain a threshold but is not a switch. The amount of activated NFAT is very small below the threshold but increases gradually above the threshold. This should be compared with the discussion in [12. Consider first the effect of varying $c$ while keeping the other parameters fixed. $\phi \rightarrow\left(1+L_{0}\right)^{-1}$ as $c \rightarrow \infty$ and $\phi \rightarrow\left(1+L_{0} \lambda^{N}\right)^{-1}$ as $c \rightarrow 0$. The quantity $\lambda$ is assumed to be greater than one in 22 and so if $N$ is large the value of $\phi$ at zero is close to zero. Consider next what happens if $N$ tends to infinity for fixed values of the other parameters. In the region where $c>\lambda k$ the inequality $\frac{k}{c}<1$ holds. Then the limit of $\phi$ as $N \rightarrow \infty$ is given by

$$
\phi_{\infty}=\left[1+L_{0} \frac{\frac{k}{c}-1}{\frac{\lambda k}{c}-1}\right]^{-1}=\frac{c-\lambda k}{c-\lambda k+L_{0}(c-k)} .
$$

When $\lambda k$ tends to $c$ the function $\phi_{\infty}$ tends to zero. Let $\tilde{c}=c-\lambda k$. Then

$$
\phi_{\infty}=\frac{\tilde{c}}{\left(1+L_{0}\right) \tilde{c}+L_{0} k(\lambda-1)}
$$

for $c>\lambda k$. In the region where $c<\lambda k$ we get $\phi_{\infty}=0$. Thus $\phi_{\infty}$ is a truncated translated Hill function with exponent one as mentioned in [12]. There is also another interesting way of passing to the limit $N \rightarrow \infty$ which is more closely related to what is done in 22 . To see this it is convenient to introduce the variable $\mu=L_{0}^{\frac{2}{N}} \lambda$. Inverting this gives $L_{0}=\left(\frac{\mu}{\lambda}\right)^{\frac{N}{2}}$. Substituting this into the expression for $\phi$ gives

$$
\phi=\left[1+\left(\frac{\lambda}{\mu}\right)^{\frac{N}{2}} \frac{\left(\frac{k}{c}\right)^{N+1}-\left(\frac{\mu}{\lambda}\right)^{N+1}}{\frac{k}{c}-\frac{\mu}{\lambda}} \frac{\frac{k}{c}-1}{\left(\frac{k}{c}\right)^{N+1}-1}\right]^{-1} .
$$

Denote the limit of this function as $\hat{\phi}_{\infty}$. Assume that $\frac{\mu}{\lambda}$ is a fixed number which is less than one. When $c>\sqrt{\frac{\lambda}{\mu}} k$ the function $\hat{\phi}_{\infty}$ is equal to one while when $c<\sqrt{\frac{\lambda}{\mu}} k$ it is equal to zero. Thus $\hat{\phi}_{\infty}$ is a translated Heaviside function and this limit behaves in a similar way to the limit of a Hill function when the exponent is allowed to tend to infinity while all other parameters are fixed. It is interesting to note that the threshold in $\hat{\phi}_{\infty}$ occurs in a different place from the threshold in $\phi_{\infty}$. In the example plotted in Fig. 2(b) of [22] the parameters are chosen as $\lambda=10$ and $\mu=1$. Then the threshold for $\hat{\phi}_{\infty}$ is at about 3.3. At the threshold value of $c$ the function $\phi$ has exactly the value one half. One key property implemented by this choice of parameters is that the reaction 
constants are such that there is a strong tendency for weakly phosphorylated NFAT to change from the inactive to the active conformation and for highly phosphorylated NFAT to change from the active to the inactive one.

The validity of the above explicit formulae is restricted to the decoupled system but they do provide some information about the full system where $d$ and $f$ are non-zero. When all other parameters are fixed there is a unique stationary solution in a given reaction simplex for each choice of non-negative values of $d$ and $f$. It can easily be shown, using the compactness of the reaction simplex, that the stationary solution depends continuously on the parameters. Since its position is known explicitly when $d=f=0$ its position is known approximately when these two parameters are small.

For any solution of the SH system let $\phi$ be the proportion of NFAT in the cytosol which is in the active state and $\psi$ the fraction of NFAT in the nucleus which is in the inactive state. Let $Z$ be the fraction of NFAT which is in the nucleus. Let $V_{1}$ and $V_{2}$ be the volume of cytosol and nucleus. Then by the conservation of the total amount of NFAT the relation

$$
f Z \psi V_{2}=d(1-Z) \phi V_{1}
$$

holds for any stationary solution and so

$$
Z=\frac{d \phi V_{1}}{d \phi V_{1}+f \psi V_{2}} .
$$

If $f$ and $d$ small then we can obtain approximate expressions for $\phi$ and $\psi$ as functions of the reaction constants. Thus a corresponding approximation is obtained for $Z$.

\section{Modelling the calcium influx}

The model presented in the last section gives a description of how the proportion $Z$ of active NFAT in the nucleus depends on the parameters describing the state of the cell. An idealized experiment would then consist in modifying the rate constants $c$ and $C$ by stimulating the cell and measuring the resulting change in $Z$. In real experiments things are more complicated. Consider now the experiments carried out in [19. There, among other things, the following type of experiment was done. A population of $\mathrm{T}$ cells was treated with different concentrations of ionomycin and the production of IL- 2 by these cells was measured. To get production of IL-2 the second signal is also required and it is produced artificially by stimulating the cells with PMA (phorbol 12-myristate 13 -acetate). The result for a given level of stimulation is a curve describing the number of cells producing different amounts of IL-2. If all the cells were identical this curve would reduce to a Dirac $\delta$ but in reality this is smeared out to a smooth curve by the natural variability of the cells. When the stimulus is varied the following phenomenon is observed. The curve in general consists of two peaks separated by a region of production rates where there are very few 
cells. As the stimulus is varied the height of one of the peaks grows while that of the other shrinks. The value at which the maximum of a peak is attained is essentially unchanged. The interpretation of this result is that a given cell either produces almost no IL-2 or produces IL-2 at a close to maximal rate. It exhibits a switch behaviour. This corresponds to the switch-like dependence of $\phi$ on $c$ found in the model.

In the analysis of these results it is supposed that the number of cells where IL-2 production is switched on closely reflects the number of cells where $Z$ is almost one. On the other hand the idea that different levels of stimulation can be modelled by taking different constant values of $c$ and $C$ may be oversimplified and this issue will now be looked at in more detail. The idealized situation would be to set a constant level of calcineurin activity by setting a constant calcium concentration. In fact it is observed experimentally that when a calcium influx is caused by stimulating the TCR and CD28, or by treating the cell with ionomycin and PMA, the concentration of calcium often displays oscillations. It is also possible that these oscillations, rather than being an unimportant side effect, encode information. A rise in calcium concentration does not only affect the NFAT signalling pathway but also other characteristics of the cell. This raises the question of how one signal can control several outputs. One possibility is that the information is encoded in the time dependence of the calcium concentrations and that the oscillations have an important role to play in this. Some experimental results on calcium oscillations in liver cells and $\mathrm{T}$ cells are described in 24 and 5 respectively. For an extensive review of modelling of calcium dynamics and signalling see 7 .

The constants $c$ and $C$ represent the concentration of active calcineurin and so in modelling the effects of calcium influx they should be replaced by functions of time. How does the concentration of active calcineurin depend on the concentration of calcium? In 22] this is modelled by an equation of the form

$$
\frac{d z}{d t}=a\left(z_{0}-z\right) y-b z
$$

where $z$ is the concentration of active calcineurin, $y$ is the concentration of calcium in the cytosol and $a$ and $b$ are constants. It remains to model the dependence of the calcium concentration on time, possibly including oscillations. In 22] this is done by means of a system of two ordinary differential equations. These equations contain the concentration of $\mathrm{IP}_{3}$. With a view to modelling stimulation by ionomycin it will be assumed that the concentration of $\mathrm{IP}_{3}$ is constant. Then the model for the calcium concentration is schematically of the following form:

$$
\begin{aligned}
& \dot{x}=\rho[-\alpha(x-y)+\beta y-\lambda f(y)(x-y)], \\
& \dot{y}=\alpha(x-y)-\beta y+\lambda f(y)(x-y)+\gamma-\delta y .
\end{aligned}
$$

Here $x$ is the concentration of calcium in the lumen of the endoplasmic reticulum. The quantities $\alpha, \beta, \gamma, \delta, \lambda$ and $\rho$ are positive constants and $f$ is a positive function describing the response of the $\mathrm{IP}_{3}$ receptor to the calcium concentration. In [22] it is chosen to be a Hill function with exponent two. This system is 
essentially a special case of one introduced by Somogyi and Stucki [24]. The only reason it is not included is that the model in [24] has $\rho=1$. Thus it implicitly assumes that the cytosol and the lumen of the endoplasmic reticulum have the same volume. In 24] another variant was also considered where the fact that $y$ is much smaller than $x$ is used to replace $x-y$ by $x$ wherever it occurs in the above system. The above system will be called the modified Somogyi-Stucki model while the other variant will be called the unmodified Somogyi-Stucki model. Note that in the unmodified system a multiplicative constant like $\rho$ can be removed by rescaling the variable $x$ and some of the parameters. It is remarked in 24 that taking $f(y)=y^{2}$ and $\alpha=0$ in the unmodified model gives a system which is equivalent to the well-known Brusselator [20].

Now some remarks will be made on the dynamics of solutions of the system (17)-(18). Note first that the only terms on the right hand side of the evolution equation for a given unknown which are negative contain a factor of that unknown. Thus it can be shown as in the proof of Lemma 1 that a solution which starts positive remains positive. Taking a linear combination of the two evolution equations shows that any stationary solution $\left(x_{*}, y_{*}\right)$ satisfies $y_{*}=\frac{\gamma}{\delta}$. Substituting this back in the equation $\dot{x}=0$ gives

$$
x_{*}=\frac{\gamma\left[\alpha+\beta+\lambda f\left(y_{*}\right)\right]}{\delta\left[\alpha+\lambda f\left(y_{*}\right)\right]} .
$$

Thus, in particular the system has exactly one stationary solution for any choice of the parameters and the function $f$. Linearizing the system about the stationary solution gives

$$
\begin{aligned}
& \frac{d \tilde{x}}{d t}=\rho\left\{\left[-\alpha-\lambda f\left(y_{*}\right)\right] \tilde{x}+\left[\alpha+\beta-\lambda f^{\prime}\left(y_{*}\right)\left(x_{*}-y_{*}\right)+\lambda f\left(y_{*}\right)\right] \tilde{y}\right\} \\
& \frac{d \tilde{y}}{d t}=\left[\alpha+\lambda f\left(y_{*}\right)\right] \tilde{x}+\left[-\alpha-\beta+\lambda f^{\prime}\left(y_{*}\right)\left(x_{*}-y_{*}\right)-\lambda f\left(y_{*}\right)-\delta\right] \tilde{y}
\end{aligned}
$$

The determinant of the linearization at $\left(x_{*}, y_{*}\right)$ is $\delta \rho\left(\alpha+\lambda f\left(y_{*}\right)\right)>0$. Thus the linearized stability of the stationary point is determined by the sign of the trace of the linearization which is given by

$$
-(1+\rho) \alpha-\beta-\delta-(1+\rho) \lambda f\left(y_{*}\right)+\lambda f^{\prime}\left(y_{*}\right)\left(x_{*}-y_{*}\right) .
$$

The stationary solution is a hyperbolic source if and only if

$$
\rho<\left(\alpha+\lambda f\left(y_{*}\right)\right)^{-1}\left[-\alpha-\beta-\delta-\lambda f\left(y_{*}\right)+\lambda f^{\prime}\left(y_{*}\right)\left(x_{*}-y_{*}\right)\right] .
$$

Since $x_{*}$ and $y_{*}$ do not depend on $\rho$ this shows that the region of instability is non-empty and is bounded by a smooth hypersurface in parameter space.

In the model of 22 the nonlinearity is given by $f(y)=\frac{y^{2}}{A+y^{2}}$. It follows that $f^{\prime}(y)=\frac{2 A y}{\left(A+y^{2}\right)^{2}}$ and so

$$
-(1+\rho) f\left(y_{*}\right)+f^{\prime}\left(y_{*}\right)\left(x_{*}-y_{*}\right)
$$




$$
\begin{aligned}
& =-(1+\rho) \frac{y_{*}^{2}}{A+y_{*}^{2}}+\frac{2 A y_{*}}{\left(A+y_{*}^{2}\right)} \frac{\beta \gamma}{\left.\delta\left[\alpha\left(A+y_{*}^{2}\right)+\lambda y_{*}^{2}\right)\right]} \\
& =\frac{\left\{-(\alpha+\lambda)(1+\rho) y_{*}^{2}+A[-(1+\rho) \alpha+2 \beta]\right\} y_{*}^{2}}{\left(A+y_{*}^{2}\right)\left[\alpha\left(A+y_{*}^{2}\right)+\lambda y_{*}^{2}\right]} .
\end{aligned}
$$

This means in particular that in this case a necessary condition for the positivity of the trace is

$$
\left(\frac{\gamma}{\delta}\right)^{2}<\frac{A[2 \beta-(1+\rho) \alpha]}{(\alpha+\lambda)(1+\rho)}
$$

Now it will be proved that every solution of the Somogyi-Stucki model is bounded. Note first that

$$
\frac{d}{d t}(x+\rho y)=\rho(\gamma-\delta y)
$$

Let $L_{C}$ be the part of the line $x+\rho y=C$ for which $y \geq \gamma / \delta$. No solution can cross it in the direction of increasing $y$. Let $L^{\prime}$ be the line $y=\frac{\gamma}{\alpha+\beta+\delta+\lambda}$. It cannot be crossed in the direction of decreasing $y$. Let $H$ be the zero set of the right hand side of (17). Its equation can be written as

$$
x=y\left[1+\frac{\beta}{\alpha+\lambda f(y)}\right] .
$$

This curve starts at the origin and as $x$ tends to infinity along it $y$ tends to infinity. Let

$$
M=\frac{\gamma}{\delta}\left[1+\frac{\beta}{\alpha+\lambda f\left(\frac{\gamma}{\delta}\right)}\right]
$$

Then the curve crosses the line $y=\gamma / \delta$ when $x=M$. Define a region $R_{C}$ to be that bounded by the $y$-axis, the line $L_{C}$, the vertical line joining the endpoint of $L_{C}$ with $L^{\prime}$ and the part of $L^{\prime}$ between its intersection with that vertical line and the $y$-axis. For $C \geq M+\frac{\rho \gamma}{\delta}$ this region is invariant under the flow. Hence any solution which starts in $R_{C}$ remains there and is bounded. Since the union of the allowed $R_{C}$ cover the region $y \geq \frac{\gamma}{\alpha+\beta+\delta+\lambda}$ it follows that any solution which tended to infinity would have to lie in the strip $y<\frac{\gamma}{\alpha+\beta+\delta+\lambda}$. Hence for a solution which is unbounded $y>M$ implies $\dot{y}<0$, a contradiction, and all solutions are bounded. It follows from Poincaré-Bendixson theory that unless the trace of the linearization at the stationary solution vanishes each solution either converges to a stationary solution or to a periodic solution. The condition on the trace is required so as to rule out possible orbits homoclinic to the stationary solution.

For certain ranges of the parameters it can be shown that there are no periodic solutions. To see this note first that any periodic solution must lie in the region $y>\frac{\gamma}{\alpha+\beta+\delta+\lambda}$. For it can neither lie entirely in the complement of that region, due to the monotonicity of $y$ there, nor leave that region. It must lie in some $R_{C}$ and in fact it must do so for some $C \leq M+\frac{\rho \gamma}{\delta}$. For the boundary of $R_{C}$ with any larger value of $C$ can only be crossed in one direction. In particular 
for this solution $x$ can never exceed $M$. Consider the divergence of the vector field defining the dynamical system. If the divergence is negative on a region containing the solution being considered then a contradiction is obtained. This divergence is given by replacing $x_{*}$ and $y_{*}$ in (22) by $x$ and $y$ respectively and can be written in the form

$$
-\left[(\rho+1) \alpha+\beta+\delta+(\rho+1) \lambda f(y)+\lambda f^{\prime}(y) y\right]+\lambda f^{\prime}(y) x .
$$

The last term is the only positive one and it can be bounded above by $\frac{3 \sqrt{3} \lambda M}{8 A^{\frac{3}{2}}}$ when $x \leq C$. Hence if

$$
\frac{3 \sqrt{3} \lambda M}{8 A^{\frac{3}{2}}} \leq(\rho+1) \alpha+\beta+\delta
$$

the divergence is negative on $R_{C}$. It follows that a sufficient condition for the absence of periodic solutions is that

$$
\frac{3 \sqrt{3} \lambda \gamma}{8 A^{\frac{3}{2}} \delta}\left(1+\frac{\beta}{\alpha+\lambda f\left(\frac{\gamma}{\delta}\right)}\right) \leq(\rho+1) \alpha+\beta+\delta .
$$

When there are no periodic solutions it can be concluded that every solution converges to the stationary solution.

In 22] numerical simulations were done for the above model with certain values of the parameters. These are:

$$
\alpha=0.001, \gamma=0.38, \delta=1, \lambda=0.16, \rho=10, A=0.25 .
$$

In two different simulations $\beta$ was chosen to be 0.1 and 1 . In both these cases the quantity (22) is negative and so the stationary solution is a hyperbolic sink. For $\beta=0.1$ the inequality (25) is violated and so this necessary condition suffices to verify that the stationary solution is a sink in that case. For $\beta$ sufficiently large with the other parameters as above the trace is positive. The value of $\beta$ for which the trace vanishes is about 8.2. For $\beta=0.1$ the inequality (31) is satisfied and the criterion for the absence of periodic solutions applies. For $\beta=1$ it does not.

Suppose that for certain values of the parameters in (17)-(18) and certain initial data the solution converges exponentially to the stationary solution $\left(x_{*}, y_{*}\right)$ as $t \rightarrow \infty$. In other words, assume there are positive constants $\eta$ and $R$ such that

$$
\left|x(t)-x_{*}\right|+\left|y(t)-y_{*}\right| \leq R e^{-\eta t}
$$

The equation (16) can be rewritten as

$$
\frac{d z}{d t}=-(b+a y) z+a z_{0} y
$$

Define

$$
z_{*}=\frac{a z_{0} y_{*}}{b+a y_{*}}
$$


Then it follows from the variation of constants formula that

$$
z(t)=z\left(t_{0}\right) e^{-b\left(t-t_{0}\right)} e^{-a \int_{t_{0}}^{t} y(s) d s}+\int_{t_{0}}^{t} e^{-b(t-s)} e^{-a \int_{s}^{t} y(u) d u} a z_{0} y(s) d s
$$

and hence that $z(t)=z_{*}+\ldots$ where the remainder decays exponentially as $t \rightarrow \infty$.

What can be said about the asymptotic behaviour of solutions of the system obtained when the SH model is generalized by replacing the constants $c$ and $C$ by functions of $t$ which converge to constants $c_{*}$ and $C_{*}$ respectively as $t \rightarrow$ $\infty$ ? Denote by $X(t)$ a solution of the system of ODE obtained from the SH model by allowing the coefficients to be time dependent. Denote this system by $\dot{X}=F(X)$. Suppose that these coefficients converge to positive limits as $t \rightarrow \infty$ and that their time derivatives tend to zero. If $t_{n}$ is a sequence tending to infinity as $n \rightarrow \infty$ consider the translates $X_{n}(t)=X\left(t+t_{n}\right)$. Because of the compactness of the reaction simplex these are uniformly bounded. Using this in the evolution equations shows that the time derivatives $X_{n}^{\prime}(t)$ are also uniformly bounded. Differentiating the equation shows that the sequence $X_{n}^{\prime \prime}(t)$ is uniformly bounded. By the Arzela-Ascoli theorem there is a subsequence $X_{n_{r}}$ which converges together with its first derivatives uniformly on compact subsets to a limit $X_{\infty}(t)$. The functions $X_{n}$ satisfy

$$
\dot{X}_{n}(t)=F\left(X_{n}(t)\right)=F\left(X\left(t+t_{n}\right)\right)=F_{\infty}\left(X_{n}(t)\right)+\ldots .
$$

Here $F_{\infty}$ is obtained by replacing the coefficients in $F$ by their limiting values and the remainder term converges to zero as $n \rightarrow \infty$. Hence

$$
\dot{X}_{\infty}(t)=F_{\infty}\left(X_{\infty}(t)\right)
$$

and $X_{\infty}(t)$ solves the SH system. All solutions of the SH system tend to the same limiting value $X_{*}$. Thus by passing to a subsequence once more it can be seen that each sequence $\left\{t_{n}\right\}$ has a subsequence along which $X(t)$ converges to $X_{*}$. Thus in fact $X(t) \rightarrow X_{*}$.

If a solution of (17)-(18) does not converge to a stationary solution then it must converge to a periodic solution. Moreover, there are solutions where this happens whenever the parameters are such that the stationary point $\left(x_{*}, y_{*}\right)$ is unstable. In this case it is more difficult to understand the behaviour of $z$ and of the modified version of the $\mathrm{SH}$ model with variable $c$ and $C$.

\section{Conclusions and outlook}

In this paper the properties of some mathematical models of parts of the NFAT signalling pathway have been investigated. One of the main results concerns a model describing the phosphorylation states of the transcription factor NFAT and the transport of these between the cytosol and the nucleus. Using chemical reaction network theory it was shown that for any value of the parameters in this system every solution converges to a stationary solution as $t \rightarrow \infty$ and that 
this stationary solution is uniquely determined by the total amount of NFAT in the cell. Moreover it was shown that in the case where transport between the two compartments is slow compared to the reactions within each compartment the stationary solution can be approximated by explicit expressions derived in 22]. It was exhibited in which way this model can behave as a switch.

The parameters in the model describing the phosphorylation states include some which reflect the concentration of active calcineurin in the cytosol. It is a priori not clear that it is sufficient to assume that this concentration is constant and for this reason a model allowing a time-dependent calcineurin concentration was examined. The concentration of active calcineurin depends on the calcium concentration in the cytosol and this concentration is affected when a $\mathrm{T}$ cell is activated. The core model studied is a two-dimensional dynamical system closely related to a model for calcium dynamics introduced in 24. Solutions of this model become periodic at late times for some values of the parameters and converge to a constant at late times for others. Criteria were obtained for the parameter values leading to these two different outcomes. It was shown that when the solution converges to a constant at late times this leads to a calcineurin concentration which also becomes constant and that the resultant densities of the phosphorylation states of NFAT in these cases are as in the case of constant calcineurin concentrations.

In the course of this work the following additional questions arose. The criteria obtained for when exactly the system (17)-(18) does or does not have periodic solutions are far from exhaustive. In particular, one question left open is if there are parameter values for which the stationary solution is stable but there nevertheless exist periodic solutions. It was shown how the SH model is affected by an asymptotically constant input. It would be interesting to know how it is affected by an asymptotically periodic input. More generally it may be asked what can be said about a possible generalization of CRNT where the reaction constants are replaced by periodic functions.

It was remarked that the results obtained for a model like the SH model, where mass action kinetics are used, may change essentially if another type of kinetics, such as Michaelis-Menten via mass action, is used. What happens when this is replaced by effective Michaelis-Menten kinetics? Here we are talking about different ways of modelling the same system of chemical reactions and it should be possible to relate them in a mathematically rigorous way.

Ultimately, in understanding $\mathrm{T}$ cell activation, the interaction of the NFAT signalling pathway with the signalling pathways leading to other important transcription factors should be understood. Is it enough, when examining the coupled network, to confine attention to stationary solutions? Or so more complicated dynamical phenomena play a role? That they may do so is suggested by the results of [6] where it is shown that for instance $\mathrm{NFAT}$ and $\mathrm{NF} \kappa \mathrm{B}$ react to time-dependent calcium signals in different ways. A first step towards doing this could be to examine the influence of the dynamical behaviour within each of the individual pathways. One dynamical system describing $\mathrm{NF} \kappa \mathrm{B}$ signalling is given in [10]. The signal pathway leading from the T cell receptor to AP-1 passes through a MAP-kinase cascade. Mathematical modelling of this pathway 
revealing switch-like behaviour has been carried out in [1. An insightful review of the possible contributions of theory and computation to the understanding of signalling pathways, with particular attention to $\mathrm{T}$ cells, has recently been given by Chakraborty and Das [3].

Acknowledgements I am grateful to Ria Baumgrass and Bernold Fiedler for helpful discussions.

\section{A Remarks on the model of Fisher et. al.}

The purpose of this appendix is to discuss the relation of the $\mathrm{SH}$ model discussed in the main part of the text to a model introduced by Fisher et. al. in 10. The building blocks in the latter model are NFAT and calcineurin. The NFAT may be unphosphorylated or fully phosphorylated and intermediate states are not included. The calcineurin may be active or inactive and the active form may bind to the different forms of NFAT. Concentrations of all these substances in the cytosol and the nucleus are considered and all of them may be transported between the two compartments. There are twelve concentrations in all. They satisfy a system of equations which, in neutral notation, can be written as:

$$
\begin{aligned}
& \frac{d x_{1}}{d t}=k_{1} x_{5}-k_{2} x_{1}+k_{17} \frac{v_{c}}{v_{n}} x_{2}-k_{18} x_{1}+k_{15} x_{9}-k_{16} x_{1} x_{3} \\
& \frac{d x_{2}}{d t}=k_{1} x_{6}-k_{2} x_{2}+k_{18} \frac{v_{n}}{v_{c}} x_{1}-k_{17} x_{2}+k_{15} x_{10}-k_{16} x_{2} x_{4} \\
& \frac{d x_{3}}{d t}=-k_{11} x_{5} x_{3}+k_{12} x_{7}+k_{5} \frac{v_{c}}{v_{n}} x_{4}-k_{6} x_{3}+k_{15} x_{9}-k_{16} x_{3} x_{1}+k_{19} I x_{11}-k_{20} x_{3} \\
& \frac{d x_{4}}{d t}=-k_{11} x_{6} x_{4}+k_{12} x_{8}-k_{5} x_{4}+k_{6} \frac{v_{n}}{v_{c}} x_{3}+k_{15} x_{10}-k_{16} x_{4} x_{2}+k_{19} I x_{12}-k_{20} x_{4} \\
& \frac{d x_{5}}{d t}=-k_{1} x_{5}+k_{2} x_{1}-k_{4} x_{5}+k_{3} \frac{v_{c}}{v_{n}} x_{6}-k_{11} x_{5} x_{3}+k_{12} x_{7} \\
& \frac{d x_{6}}{d t}=-k_{1} x_{6}+k_{2} x_{2}-k_{3} x_{6}+k_{4} \frac{v_{n}}{v_{c}} x_{5}-k_{11} x_{6} x_{4}+k_{12} x_{8} \\
& \frac{d x_{7}}{d t}=k_{11} x_{5} x_{3}-k_{12} x_{7}+k_{7} \frac{v_{c}}{v_{n}} x_{8}-k_{8} x_{7}-k_{13} x_{7}+k_{14} x_{9} \\
& \frac{d x_{8}}{d t}=k_{11} x_{6} x_{4}-k_{12} x_{8}+k_{8} \frac{v_{n}}{v_{c}} x_{7}-k_{7} x_{8}-k_{13} x_{8}+k_{14} x_{10} \\
& \frac{d x_{9}}{d t}=k_{16} x_{1} x_{3}-k_{15} x_{9}+k_{13} x_{7}-k_{14} x_{9}+k_{9} \frac{v_{c}}{v_{n}} x_{10}-k_{10} x_{9} \\
& \frac{d x_{10}}{d t}=k_{16} x_{2} x_{4}-k_{15} x_{10}+k_{13} x_{8}-k_{14} x_{10}+k_{10} \frac{v_{n}}{v_{c}} x_{9}-k_{9} x_{10} \\
& \frac{d x_{11}}{d t}=k_{5} \frac{v_{c}}{v_{n}} x_{12}-k_{6} x_{11}-k_{19} I x_{11}+k_{20} x_{3} \\
& \frac{d x_{12}}{d t}=-k_{5} x_{12}+k_{6} \frac{v_{n}}{v_{c}} x_{11}-k_{19} I x_{12}+k_{20} x_{4}
\end{aligned}
$$


Here all quantities other than the unknowns $x_{i}$ are positive constants. To see the relation of these equations to those of [10] it suffices to note that they are written in the same order in both cases. There are conserved quantities

$$
J_{1}=v_{n}\left(x_{1}+x_{5}+x_{7}+x_{9}\right)+v_{c}\left(x_{2}+x_{6}+x_{8}+x_{10}\right) .
$$

and

$$
J_{2}=v_{n}\left(x_{3}+x_{7}+x_{9}+x_{11}\right)+v_{c}\left(x_{4}+x_{8}+x_{10}+x_{12}\right) .
$$

$J_{1}$ is the total concentration of NFAT in all forms and $J_{2}$ the total concentration of calcineurin.

These equations do not appear to incorporate any switch-like mechanism and so it is difficult to see how they could model the behaviour observed in [19]. The network structure contains rather little information about the nature of the substances involved and so it is to be expected that important information influencing the dynamics is encoded in the numerical values of the reaction constants $k_{i}$. For instance, the fact that the central role of calcineurin in this context is that of a phosphatase is not visible from the equations. In [10] specific experimentally motivated values are chosen for the $k_{i}$ and the character of calcineurin as a phosphatase is encoded in the fact that $k_{13}$ is much greater than $k_{14}$. In view of this it is perhaps not surprising that CRNT is not too helpful in this case. There are sixteen complexes and only one linkage class. Since the rank of $\bar{N}$ cannot be more than the number of species, which is twelve, the deficiency must be at least three. Thus this system is far from the low deficiency situation where CRNT is most powerful. Nevertheless a couple of simple conclusions can be drawn.

Because of the conserved quantities the reaction simplex is compact and all solutions exist globally in time. Suppose that a solution with $J_{1}>0$ and $J_{2}>0$ has an $\omega$-limit point on the boundary of the positive quadrant. Then arguing as in Section 2 we can conclude that there is a solution $c_{*}$ lying in the boundary and that at late times it has a constant number of non-zero components. Let $n_{1}$ be the number of non-zero components and $n_{0}=n-n_{1}$. Let $\mathcal{S}_{1}$ and $\mathcal{S}_{0}$ be the corresponding subsets of $\mathcal{S}$. It is impossible that there is a reaction whose left hand side is a species belonging to $\mathcal{S}_{1}$ and whose right hand side includes a species belonging to $\mathcal{S}_{0}$. Inspecting the pairs of species where there are reactions of this type in both directions shows that each of the following three sets are subsets of either $\mathcal{S}_{0}$ or $\mathcal{S}_{1}$

$$
\mathcal{S}_{1}^{\prime}=\left\{x_{1}, x_{2}, x_{5}, x_{6}\right\}, \mathcal{S}_{2}^{\prime}=\left\{x_{3}, x_{4}, x_{11}, x_{12}\right\}, \mathcal{S}_{3}^{\prime}=\left\{x_{7}, x_{8}, x_{9}, x_{10}\right\} .
$$

Moreover if $\mathcal{S}_{3}^{\prime} \subset \mathcal{S}_{1}$ then $\mathcal{S}_{1}=\mathcal{S}$. This possibility is ruled out by the fact that the solution lies on the boundary of the positive orthant. Inspecting the system once again and using the form of the nonlinear terms shows that if both $\mathcal{S}_{1}^{\prime}$ and $\mathcal{S}_{2}^{\prime}$ are subsets of $\mathcal{S}_{1}$ a contradiction is obtained. Thus the only remaining possibilities are $\mathcal{S}_{1}=\mathcal{S}_{1}^{\prime}$ of $\mathcal{S}_{1}=\mathcal{S}_{2}^{\prime}$. However each of these implies the vanishing of one of the conserved quantities. It can be concluded that a solution for which $Z_{1}$ and $Z_{2}$ are non-zero has no $\omega$-limit points on the boundary. 
It follows from the Brouwer fixed point theorerm as in Theorem I.8.2 of [13] that there exists a stationary solution in the reaction simplex. Since it has been shown that this solution does not lie on the boundary it must lie in the interior. Thus this system has at least one positive stationary solution for any choice of the parameters and any biologically relevant initial data. On the other hand this argument gives no indication as to whether there might be multiple stationary solutions or more complicated dynamics such as periodic solutions.

\section{References}

[1] Altan-Bonnet G. and Germain, R. N. 2005 Modeling T cell antigen discrimination based on feedback control of digital ERK responses. PloS Biol. 3 (11): e356.

[2] Anderson, D. 2011 A proof of the global attractor conjecture in the single linkage class case. SIAM J. Appl. Math. 71, 1487-1508.

[3] Chakraborty, A. K. and Das, J. 2010 Pairing computation with experimentation: a powerful coupling for understanding T cell signalling. Nature Rev. Immunol. 10, 59-71.

[4] Craciun, G., Dickenstein, A., Shiu, A. and Sturmfels, B. 2009 Toric dynamical systems. J. Symb. Comp. 44, 1551-1565.

[5] Dolmetsch, R. E. and Lewis, R. S. 1994 Signalling between intracellular $\mathrm{Ca}^{2+}$ stores and depletion-activated $\mathrm{Ca}^{2+}$ channels generates $\mathrm{Ca}^{2+}$ oscillations in T lymphocytes. J. Gen. Physiol. 103, 365-388.

[6] Dolmetsch, R. E., Lewis, R. S., Goodnow, C. G. and Healy, J. I. 1997 Differential activation of transcription factors induced by $\mathrm{Ca}^{2+}$ response amplitude and duration. Nature 386, 855-858.

[7] Falcke, M. 2004 Reading the patterns in living cells - the physics of $\mathrm{Ca}^{2+}$ signalling. Adv. Phys. 53, 255-440.

[8] Feinberg, M. 1972 Complex balancing in general kinetic systems. Arch. Rat. Mech. Anal. 49, 187-194.

[9] Feinberg. M. 1980 Lectures on chemical reaction networks. Available at http://www.chbmeng.ohio-state.edu/ feinberg/research/

[10] Fisher, W. G., Yang, P.-C., Medikonduri, R. K. and Jafri, M. S. 2006 NFAT and $\mathrm{NF} \kappa \mathrm{B}$ activation in $\mathrm{T}$ lymphocytes: a model of differential activation of gene expression. Ann. Biomed. Eng. 34, 1712-1728.

[11] Grimbs, S., Arnold, A., Koseska, A., Kurths, J., Selbig, J. and Nikoloski, Z. 2011 Spatiotemporal dynamics of the Calvin cycle: multistationarity and symmetry breaking instabilities. Biosystems 103, 212-223. 
[12] Gunawardena, J. 2005 Multisite protein phosphorylation makes a good threshold but can be a poor switch. Proc. Nat. Acad. Sci. USA 102, 1461714622 .

[13] Hale, J. K. 1969 Ordinary differential equations. Wiley Interscience, New York.

[14] Horn, F. and Jackson, R. 1972 General mass action kinetics. Arch. Rat. Mech. Anal. 47, 81-116.

[15] Horn, F. 1972 Necessary and sufficient conditions for complex balancing in chemical kinetics. Arch. Rat. Mech. Anal. 49, 172-186.

[16] McKeithan, T. W. 1995 Kinetic proofreading in T-cell receptor signal transduction. Proc. Nat. Acad. Sci. USA 92, 5042-5046.

[17] Murphy, K. M., Travers, P. and Walport, M. 2007 Janeway's immunobiology. Garland Science, New York.

[18] Murray, J. D. 1989 Mathematical biology. Springer, Berlin.

[19] Podtschaske, M., Benary, U., Zwinger, S., Höfer, T., Radbruch, A. and Baumgrass, R. 2007 Digital NFATc2 activation per cell transforms graded $\mathrm{T}$ cell receptor activation into an all-or-none IL-2 expression. PloS ONE 2(9): e935.

[20] Prigogine, I. and Lefever, R. 1968 Symmetry-breaking instabilities in dissipative systems. J. Chem. Phys. 48, 1695-1699.

[21] Roitt, I, Brostoff, J., Male, D. and Roth, D. 2006 Immunology. Mosby, New York.

[22] Salazar, C. and Höfer, T. 2003 Allosteric regulation of the transcription factor NFAT1 by multiple phosphorylation sites: a mathematical analysis. J. Mol. Biol. 327, 31-45.

[23] Salazar, C. and Höfer, T. 2009 Multisite protein phosphorylation from molecular mechanisms to kinetic models. FEBS J. 276, 3177-3198.

[24] Somogyi, R. and Stucki, J. W. 1991 Hormone-induced calcium oscillations in liver cells can be explained by a simple one pool model. J. Biol. Chem. 266, 11068-11077.

[25] Sontag, E. D. 2001 Structure and stability of certain chemical networks and applications to the kinetic proofreading model of T-cell receptor signal transduction. IEEE Trans. Automat. Contr. 46, 1028-1047

[26] Wang, L. and Sontag, E. D. 2008 On the number of steady states in a multiple futile cycle. J. Math. Biol. 57: 29-52. 